\title{
Hochverehrter, lieber Herr Professor Herzfeld!
}

Mit dieser Auswahl aus Ihren Aufsätzen möchten Freunde und Schüler Ihnen zum 70. Geburtstag ihre Verehrung und Dankbarkeit bezeugen. Die hier vereinigten Arbeiten, die als kleiner Ausschnitt aus der Fülle Ihres bisherigen Schaffens die ganze Breite und Farbigkeit Ihrer Palette nur anzudeuten vermögen, stellen nicht nur ein Stück Ihrer geistigen Biographie dar, sondern erlauben zugleich einen unmittelbaren Einblick in die Situation und die Probleme der deutschen Geschichtsforschung, mit deren innerem Leben in den Weimarer Jahren und der Zeit nach 1945 Sie unlöslich verbunden waren und sind.

Es verstand sich deshalb von selbst, daß wir zur Bewahrung der Frische und des historischen Quellencharakters Ihrer so häufig aus dem Wurzelboden der eigenen Gegenwart gespeisten Arbeiten keinerlei sachliche Änderungen durch Streichungen oder Ergänzungen vorgenommen haben. Dabei wurde bewußt in Kauf genommen, daß einige der Aufsätze Ihren heutigen Standpunkt zu einer Frage nicht mehr korrekt widerspiegeln, haben doch gerade Sie immer wieder den Mut besessen, aus der Entwicklung der Forschung zu lernen, sich selbst zu korrigieren und sich zu dem Wandel Ihrer Auffassungen auch offen zu bekennen.

Das einigende Band der hier abgedruckten Arbeiten ist das unbedingte Streben nach historischer Wahrheit, die Tiefe der Fragestellung, die Einordnung der behandelten Fragen in die historische Gesamtentwicklung, die Offenheit gegenüber neuen Anregungen, von welcher Seite auch immer, und vor allem die Ernsthaftigkeit des Ringens mit der eigenen Gegenwart, deren Probleme immer wieder Ihre historische Fragestellung bestimmen.

Wir haben aus Ihren verschiedenen Hauptarbeitsbereichen eine Auswahl getroffen, wobei wir Arbeiten zur Historiographie - ein zentrales Thema Ihrer Forschungen - an den Anfang stellten.

Die folgenden Arbeiten über Persönlichkeiten der Geschichte sind trotz ihrer weit auseinanderliegenden Entstehungszeit und der verschiedenen Lebenskreise der behandelten Gestalten durch das Interesse am Menschen, das Sie so häufig in Ihrer wissenschaftlichen Arbeit die Form der Biographie wählen läßt, innerlich verbunden. 
In dem dritten Teil über Fragen der Großen Politik haben wir einen typischen Aufsatz aus der Vielzahl Ihrer Arbeiten der Hallenser Schaffensperiode zur Vorgeschichte des Weltkrieges und zur Außenpolitik I87I-1914 sowie einige Arbeiten ausgewählt, in denen Sie in einer für Sie charakteristischen Weise, von der weitausholenden Erörterung historischer Probleme ausgehend, an die drängenden Fragen unserer Gegenwart heranführen. In diesen Aufsätzen wie in Ihrer die Forschung so stark befruchtenden Studie über das Problem des deutschen Heeres und Threr Darstellung der "Modernen Welt" wird besonders deutlich, wie die Vereinigung von "offener Revisionsbereitschaft gegenüber einer krisenhaften Gegenwart" mit „entschiedener Verwurzelung im Erdreich gewachsener Geschichte", die Sie an Näfs "Epochen der neueren Geschichte" hervor-

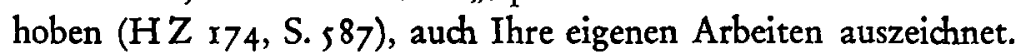

An den Schluß des Bandes haben wir einige Ihrer jüngeren Aufsätze zur Berliner Geschichte gestellt, in denen sich Ihre engen Beziehungen zu Ihrer heutigen Wirkungsstätte, Ihr seit der Beschäftigung mit dem ersten Band des Miquel nie wieder abreißendes Interesse für die Kommunalwissenschaften und Thre intensive kritische Anteilnahme an den bewegenden politischen Problemen der Zeit nach 1945 widerspiegeln.

Der Kenner Ihrer Arbeiten wird besonders die in der Zeit Ihrer Freiburger Professur für westeuropäische Geschichte entstandenen Untersuchungen über Probleme der Geschichte außerdeutscher Länder vermissen, die wegen der Beschränkung des zur Verfügung stehenden Raumes nicht aufgenommen werden konnten, obwohl sie die Weite Threr Interessen und Ihres Arbeitsfeldes sowie die Fülle der von Thren Forschungen ausgehenden Anregungen besonders deutlich gemacht hätten.

Wir sind jedoch besonders glücklich, daß wir in diesem Band zwei neue Aufsätze abdrucken können: Eine Skizze über Friedrich Meinecke als Politiker und Mensch, die auf seinem soeben erschienenen Briefwechsel beruht, und einen Versuch, die Diskussion über die Frage des deutschen Militarismus durch den Vergleich mit ausländischen Verhältnissen auf eine neue Ebene zu heben.

Wir haben lange geschwankt, ob wir das hier vorliegende, so ausführliche Verzeichnis Ihrer Schriften, Aufsätze, Rezensionen etc. oder nur eine Auswahlbibliographie Ihrer wichtigsten Arbeiten veröffentlichen sollten. Wir waren uns darüber klar, daß Sie den Druck einer vollständigen Bibliographie nie zugelassen hätten, da es Ihnen in Ihrer Bescheidenheit zutiefst zuwider gewesen wäre, so viel Aufhebens von sich selbst zu machen. 
Neben dem Unbehagen, das wohl jeder Historiker angesichts von Auszügen und Kürzungen empfindet, schienen uns noch weitere schwerwiegende sachliche Erwägungen für die Veröffentlichung der von Werner Schochow in eigener Verantwortung bearbeiteten Bibliographie zu sprechen. Ihre Rezensionen waren ja nie ein Nebenprodukt Ihrer wissenschaftlichen Arbeit, sondern waren vielmehr ein Ausdruck jener wachen kritischen Beobachtung und Förderung der Forschung auf Ihren Arbeitsgebieten, die seit Thren ersten Besprechungen in der Deutschen Literaturzeitung und der Historischen Zeitschrift sowie Ihren regelmäßigen Literaturübersichten für den Zeitraum I 890-1919 in den Jahresberichten einen wesentlichen Bestandteil Ihrer Arbeiten ausmacht. Die souveräne Beherrschung Ihres Faches, von der die in kürzester Zeit verfaßte „Moderne Welt" ein eindrucksvolles Zeugnis ablegt, beruht nicht zuletzt auf dieser umfassenden und intensiven Rezensionstätigkeit und Ihrer Praxis, unabhängig von den jeweils vorliegenden eigenen Forschungsprojekten die gesamte wichtige Literatur auf Ihrem Lehrgebiet und dessen Randgebieten $\mathrm{zu}$ verarbeiten.

Als Sie am I. 5. I950 auf Wunsch von Friedrich Meinecke auf das Ordinariat für Neuere Geschichte an der Freien Universität berufen wurden, lagen hinter Ihnen die Jahre des seit 1938 erzwungenen Schweigens, der Verfolgung und der im Vergleich zu Ihrer späteren Berliner Arbeit beschaulichen Freiburger Lehrtätigkeit nach 1946, die in der liebevollen, behutsamen Skizze Ihres Lebensweges von Carl Hinrichs nachgezeichnet wurden ${ }^{1}$. Uns allen will es oft so erscheinen, als ob nun in diesem 1950 einsetzenden neuen Lebensabschnitt, in dem Sie noch mitten drinstehen, die in der erzwungenen Muße aufgespeicherte Arbeitskraft und der gestaute Gestaltungsdrang Ihrer so lebensvollen Persönlichkeit zur Entladung drängte. Die entscheidende Mitwirkung am Aufbau des FriedrichMeinecke-Institutes und auch der Freien Universität, die schöpferische Initiative in Fragen der Universitätsreform, die Schaffung eines Institutes für Berliner Zeitgeschichte, die Wirksamkeit als Vorsitzender der Berliner Historischen Kommission, die aktive Beteiligung an der Entwicklung und Arbeit der Berliner Lehr- und Forschungsinstitute für politische Wissenschaften, die Förderung der Kommunalwissenschaften, die Mitarbeit an den internationalen Vereinbarungen über die Gestaltung von Schulbüchern,

1 Zwei Berliner Historiker. In: Jahrbuch für die Geschichte Mittel- u. Ostdeutschlands 6 (1957), S. x ff. 
die tätige Mitgliedschaft in einer Reihe weiterer wissenschaftlicher Institutionen und Ihre nie abreißende Vortragsarbeit sind ein Ausdruck dieser erstaunlichen und bewundernswerten Aktivität.

Trotzdemaber sind Sie nicht im Managertum aufgegangen, sondern haben in der oft über den wissenschaftlichen Bereich hinausgehenden Beratung und Förderung Ihrer Studenten den Kern Ihrer Lebensaufgabe gesehen.

Das Geheimnis Ihres so großen Erfolges als akademischer Lehrer, von dem das Verzeichnis Ihrer Schüler zeugt, liegt nicht darin, daß Sie versucht hätten, Ihre Studenten suggestiv in Ihren Bann zu ziehen. Es beruht auch nicht darauf, daß Sie die Aufarbeitung des Quellenmaterials zu einem Sie interessierenden größeren Forschungsproblem in Form von Einzeluntersuchungen an Ihre Schüler mit einer genauen wissenschaftlichen Marschroute weitergegeben hätten. Ein derartiges Schülerverhältnis, das das geistige Gespräch in kritikloser Anbetung erstarren ließe oder in dem das nackte Forschungsergebnis und der Doktortitel das einzige Resultat der jahrelangen Arbeit eines Doktoranden gewesen wären, hätte zu Ihrer Auffassung von der pädagogischen Pflicht eines Hochschullehrers in unüberbrückbarem Widerspruch gestanden. Es ist daher auch kein Zufall, daß Sie zwar eine große Anzahl von Schülern haben, deren Arbeiten schon in der Themenstellung Ihre Anregungen oft deutlich widerspiegeln, daß Sie aber eine Schule im engeren Sinne, in der die Handschrift des Meisters nachgeahmt wird, nicht begründet haben.

Ihre vornehmste Aufgabe sahen Sie vielmehr in der Entwicklung der Individualität und der Selbständigkeit Ihrer Schüler. Indem Sie zu neuen Ideen und Fragestellungen ermutigten, die tastenden und oft verschuïteten eigenen Ansätze Ihrer Studenten geduldig und behutsam zu klären und freizulegen versuchten und sie an einem langen, kaum noch spürbaren Leitseil führten, wobei Sie Irrwege und Umwege als das Lehrgeld eines Anfängers bewußt in Kauf nahmen, haben Sie geholfen, daß sich Ihre Schüler wissenschaftlich freischwammen. Man kann auch nicht von Ihnen als einem akademischen Lehrer sprechen, ohne Ihre souveräne, auf Ihrer absoluten inneren Sicherheit beruhende Toleranz zu erwähnen, die Sie immer wieder dazu führt, sich gerade über die von Ihren eigenen Auffassungen abweichenden, aber begründeten Meinungen Ihrer Schüler als ein Zeichen der geistigen Selbständigkeit zu freuen.

Wenn Sie daher vor wenigen Monaten auf einem Treffen ehemaliger Tutoren des Friedrich-Meinecke-Institutes von der „lebendigen Ren- 
dite" Ihrer Schüler sprachen, die Ihnen im Innersten viel mehr Befriedigung gäbe als der Ertrag Ihrer Forschungen, so lag in dieser für Sie so kennzeichnenden Formulierung, in der überströmende menschliche Wärme durch einen dem Wirtschaftsleben entnommenen Begriff mühsam zurückgedrängt wird, Ihre Freude an der Bildung der Persönlichkeit, aber auch Ihr Glaube an das ungebrochene innere Leben unserer so alten, aber doch immer neu erregenden Wissenschaft.

Ihre Freunde und Schüler, in deren Kreis die Idee zu der Aufsatzsammlung entstanden ist und die freudig die bei der Drucklegung anfallenden Arbeiten übernommen haben, verbinden mit den besten Wünschen für weitere fruchtbare Arbeit die Hoffnung auf eine lange Fortdauer jenes zur Berliner Tradition gehörenden wissenschaftlichen Gesprächs, aus dem Sie nicht fortzudenken sind.

Gerhard A. Ritter 
